\title{
Helmholtz Resonator Liner with Flexible Walls
}

\author{
Karsten Knobloch*, Lars Enghardt†, and Friedrich Bake ${ }^{\ddagger}$ \\ DLR Engine Acoustics, Institute of Propulsion Technology, German Aerospace Center, 10623 Berlin, GERMANY
}

\section{Nomenclature}

\begin{tabular}{|c|c|c|}
\hline$A$ & (duct) cross area $\left[\mathrm{m}^{2}\right]$ & reflection coefficient (amplitude)[-] \\
\hline$c$ & speed of sound $[\mathrm{m} / \mathrm{s}]$ & reflection coefficient (energy) [-] \\
\hline$f$ & frequency $[\mathrm{Hz}]$ & transmission coefficient (amplitude) [- \\
\hline$I$ & acoustic energy flux $\left[\mathrm{W} / \mathrm{m}^{2}\right]$ & transmission coefficient (energy) [-] \\
\hline$k$ & wave number $[1 / \mathrm{m}]$ & phase difference $\left[^{\circ}\right]$ \\
\hline$M$ & Mach number[-] & dissipation coefficient (energy) [-] \\
\hline$P$ & acoustic power $[\mathrm{W}]$ & $\rho \quad$ density $\left[\mathrm{kg} / \mathrm{m}^{3}\right]$ \\
\hline \multicolumn{2}{|c|}{ Subscript } & Superscript \\
\hline 1 & upstream conditions & downstream propagating \\
\hline 2 & downstream conditions & upstream propagating \\
\hline$a$ & excitation speaker A & \\
\hline$b$ & excitation speaker B & \\
\hline \multicolumn{3}{|c|}{ Abbreviations } \\
\hline DDOF & double degree of freedom (liner) & \\
\hline DS & upstream (cell) & \\
\hline SDOF & single degree of freedom (liner) & \\
\hline SPL & sound pressure level $[\mathrm{dB}]$ & \\
\hline TPU & thermoplastic polyurethane & \\
\hline US & upstream (cell) & \\
\hline
\end{tabular}

\section{Introduction}

Liners are part of every modern commercial aero-engine. Usually, they are installed in the engine intake; but also in the bypass duct or in the outlet some liners can be found. Despite the decrease of overall engine noise due to the increase of bypass-ratio (BPR), cut-off design for rotor-stator combinations, and various other means, there is an increasing demand for efficient broad-band noise absorption with the final goal of further overall noise reduction. This demand is mainly caused through the reduction of the dominating tonal components, but might be also connected to an increase in broadband noise itself. In addition, the increase in BPR requires shorter nacelles in order to reduce associated drag and weight penalties. This leads not necessarily to a smaller area for liner installation e.g. in the intake of the engine, but to a shorter length of the intake and thereby to a shorter propagation distance of emitted noise over a lined surface in axial direction. State of the art for inlet liners are single- and double-degree of freedom (SDOF and DDOF) liners consisting of cells of fixed size (for DDOF for instance with a septum dividing the individual cells) covered with a perforated face sheet, and a rigid back plate. The whole liner structure must be very robust, but at the same time of light weight, withstand various fluids and environmental conditions etc. Current liners are

*Scientist, AIAA Member, correspondence to: karsten.knobloch@dlr.de

†Professor at TU Berlin and head of DLR department "Engine Acoustics", AIAA Member

${ }^{\ddagger}$ Scientist, AIAA Member 
therefore made of glass-fiber and carbon fiber reinforced composite structures with the separating walls of the individual cells being for instance manufactured by epoxy-covered aramide paper.

A single cell or an array of identical cells of a SDOF liner can be modeled with some simplifications as a Helmholtz-resonator with cavity volume, open area of the face sheet, and thickness of the face sheet being the key dimensions for its description.

Beside the afore-mentioned reasons for efficient broadband noise damping, there is also a need for efficient damping at lower frequencies, which is usually limited by the available space (specifically depth) for the liner installation which limits the cell size.

The novel liner concept presented here relies on the interaction of the sound field with flexible walls within an otherwise classical liner cavity structure for an enhanced acoustic damping performance. Hereby, the deflection of the cell walls and intrinsic damping of the wall material shall lead to an improved damping characteristics in terms of overall absorption (dissipation) of acoustic energy and shall enlarge the frequency range of significant absorption. This concept is called "FlexiS" (Flexible Structure).

\section{Liner Design}

The FlexiS liner utilized in this investigation are designed to be tested in the aeracoustic DUCT windtunnel. The desired plane wave excitation and the duct dimensions determine the cell size and perforation of the face sheet. All liners have exactly the same cell dimensions and share the same back and cover plate design. Only on the internal cell walls in spanwise directions, a large part of these cell wall is covered with a flexible sheet material.

For comparison, an identical sample with stiff internal cell walls is build (called "REFERENCE") and tested under the same conditions as the FlexiS liners.

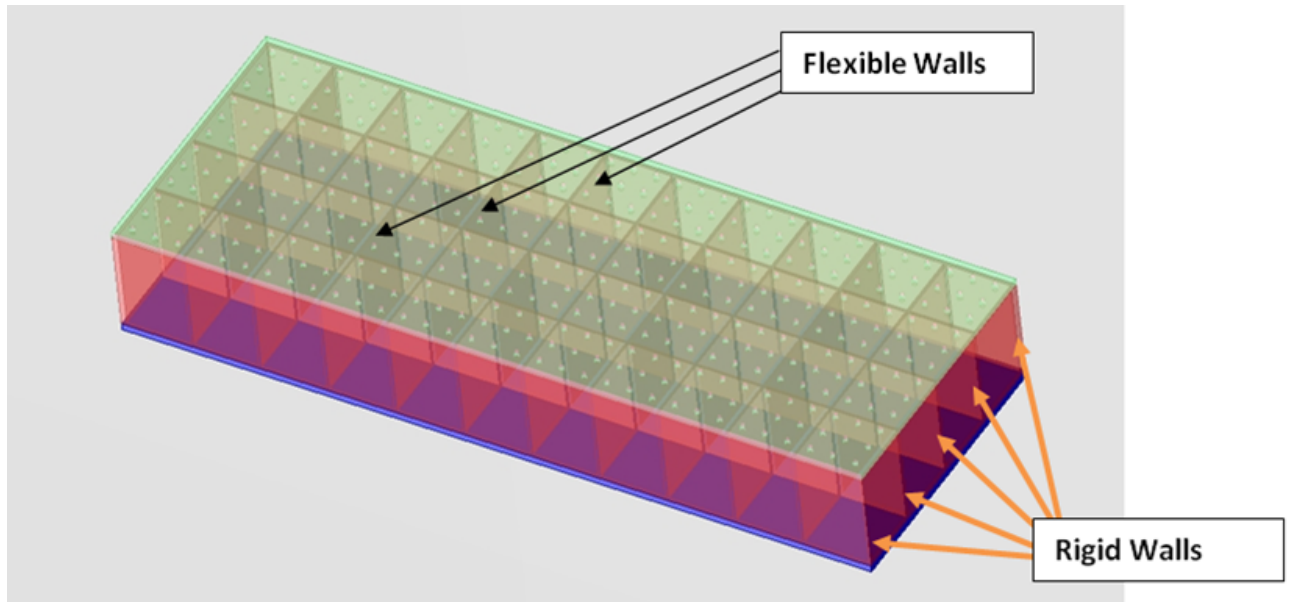

Figure 1. Principle setup of FlexiS liner concept with partially flexible walls in the liner cavity structure.

All samples consist of four by ten liner cavities in spanwise and mean flow direction, respectively. These cavities are $19 \mathrm{~mm}$ by $19 \mathrm{~mm}$ in width and length and $30 \mathrm{~mm}$ in depth. The cavities are covered with a perforated face-sheet which constitutes the interface to the duct of the wind tunnel. The perforation is setup in a way that each cavity is provided with nine holes of $1.3 \mathrm{~mm}$ diameter. With the simple formula building on Helmholtz' original work, ${ }^{1}$

$$
f_{h}=\frac{c}{2 \pi} \sqrt{\frac{A_{o}}{V\left(l_{H}+\pi r_{H} / 2\right)}}
$$

where $c$ is the speed of sound, $A_{o}$ the open area of the perforation, $V$ the volume of the cavity, $l_{H}$ the height of the perforation hole, and $r_{H}$ its radius, a resonance frequency of $1040 \mathrm{~Hz}$ can be estimated assuming that the area of the nine holes is summed up to a single opening of the equivalent cross section. 


\section{Material}

The material for the flexible walls should exhibit intrinsic (material inherent) damping in order to dissipate acoustic energy in addition to the energy conversion which takes place at the resonator facing sheet. Therefore, two different types of materials where chosen for the first experiments.

Thermoplastic-polyurethan (TPU) is easily available as sheets in various thicknesses ranging from one tenth of a millimeter to several millimeters. Material parameters can be obtained from material data sheets (e.g. from $\mathrm{BASF}^{2}$ ) or determined with specific instruments. The dynamic mechanical analysis (DMA) for instance yields the phase lag between stress and strain for a small torsional (or otherwise) deflection. The tangent of this phase lag is called the loss factor $\tan (\delta)$ or $\tan (\Delta)$ which describes the material inherent damping ( $\Delta$ not to be confused with the dissipation of acoustic energy as described in sec. B). For the materials considered here - TPU sheets of type $1195 \mathrm{~A}$ in thickness $0.1 \mathrm{~mm}$ and $0.5 \mathrm{~mm}$, and type $1170 \mathrm{~A}$ in thickness $0.3 \mathrm{~mm}$ - the loss factor is in the range of 0.05 to 0.3 . Generally, the loss factor is strongly depending on temperature and and to a certain extent also on the frequency of deflection.

A custom made sheet composed of epoxy resin in combination with polyetheramine was chosen as the second material with the emphasis here on a higher loss factor compared to the commercially available TPU sheets. It has a thickness of $0.5 \mathrm{~mm}$ and is identified in the results section as "EP-0.5".

\section{Acoustic Tests}

In order to assess the acoustic performance, the liners were fitted in the measurement section of the DUct aCoustic Test rig - Rectangular cross section (DUCT-R) facility of the German Aerospace Center (DLR) in Berlin. The rig has a cross section of $60 \mathrm{~mm} \times 80 \mathrm{~mm}$, a length of about $8 \mathrm{~m}$ and consists of two symmetrical parts, which are mounted upstream and downstream of the tested liner (see sketch in Figure 2). A radial compressor is connected to the upstream part of the duct via a tube system to provide a grazing flow. A maximum Mach number of about 0.3 can be achieved at the duct centerline. The duct has an overall number of 106 microphone positions which are spread over the rig as described by Busse-Gerstengarbe et al. ${ }^{3}$ For the current tests, only 10 microphones were mounted.

The rig has been used for the investigation of scattering coefficients and impedances of several liner types during the last years. It is known to have a very low error in derived dissipation (1-3\%, see e.g. Lahiri $\left.{ }^{4}\right)$.

\section{A. Experimental Setup}

The basic setup for the acoustic measurements is identical to the setup for a conventional HelmholtzResonator liner (SDOF) liner. By the upstream and downstream speakers, a multitone is excited and fed into the rig. Consecutively, six multi-tones covering the frequency range from 204 to $2091 \mathrm{~Hz}$ with a $51 \mathrm{~Hz}$ resolution are used. The overall SPL of these multi-tones was adjusted to about $130 \mathrm{~dB}$, but an identical SPL for all individual frequency components could not be fully ensured due the speaker characteristics and the acoustic coupling of the speaker to the duct. The cut-on frequency for higher-order modes in the DUCT-R rig is about $2150 \mathrm{~Hz}$ for the no-flow case.

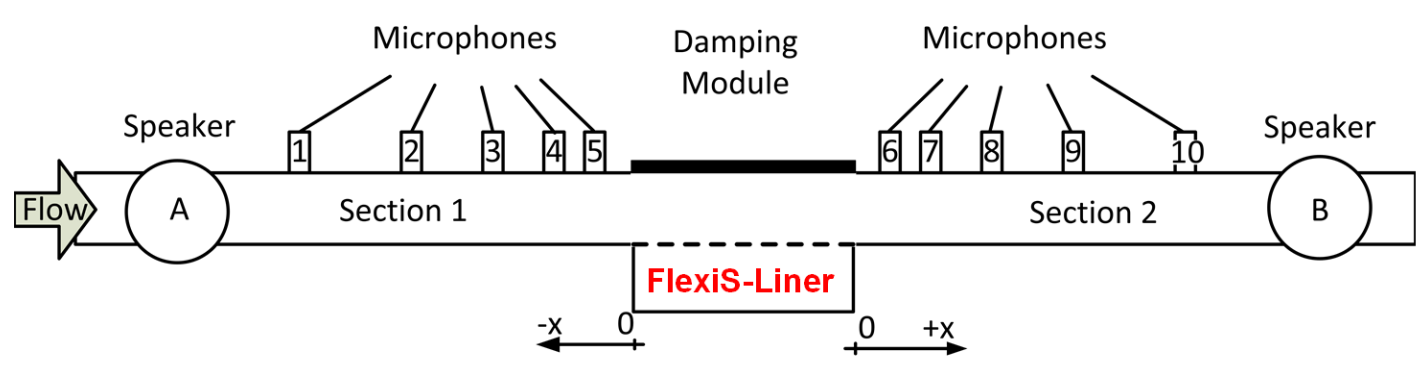

Figure 2. Schematic view of the DUCT test rig. 


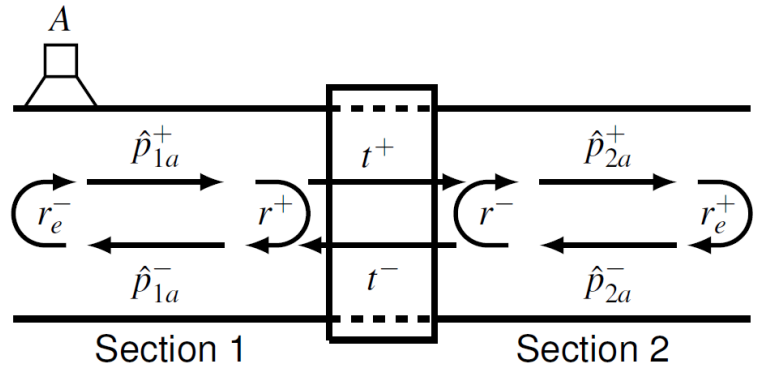

(a) MEASUREMENT $A$

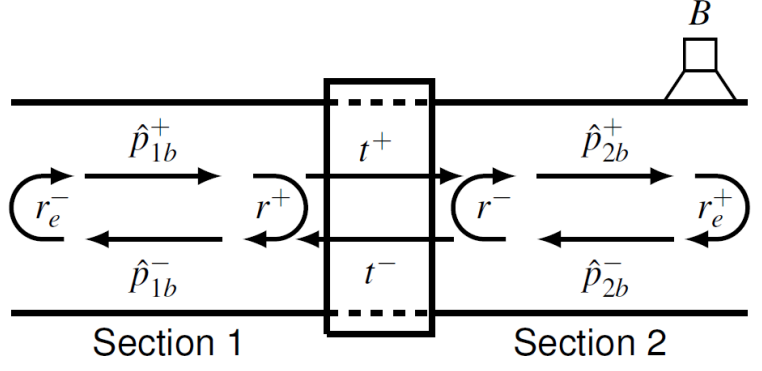

(b) MEASUREMENT $B$

Figure 3. Illustration of the sound field in the duct for measurements $A$ and $B$ by means of the sound pressure amplitudes $\hat{p}$, the reflection coefficient $r$, the transmission coefficient $t$, and the end reflection $r_{e}$.

\section{B. Data Processing}

For each liner test configuration two different sound fields are excited consecutively in two separate measurements (index a and b). Speaker A is used in the first measurement and in the second measurement the same signal is fed into speaker B. Then, the data of section 1 and section 2 (index 1 and 2) are analyzed separately. This results in four equations for the complex sound pressure amplitudes for each section and measurement:

$$
\begin{aligned}
\hat{p}_{1 a}(x) & =\hat{p}_{1 a}^{+} e^{-i k_{1}^{+} x}+\hat{p}_{1 a}^{-} e^{i k_{1}^{-} x} \\
\hat{p}_{2 a}(x) & =\hat{p}_{2 a}^{+} e^{-i k_{2}^{+} x}+\hat{p}_{2 a}^{-} e^{i k_{2}^{-} x} \\
\hat{p}_{1 b}(x) & =\hat{p}_{1 b}^{+} e^{-i k_{1}^{+} x}+\hat{p}_{1 b}^{-} e^{i k_{1}^{-} x} \\
\hat{p}_{2 b}(x) & =\hat{p}_{2 b}^{+} e^{-i k_{2}^{+} x}+\hat{p}_{2 b}^{-} e^{i k_{2}^{-} x}
\end{aligned}
$$

$\hat{p}^{+}$and $\hat{p}^{-}$are the complex amplitudes of the downstream and upstream traveling waves with their respective wave numbers $k^{ \pm}$.

The recorded microphone signals are transformed into the frequency domain using the method presented by Chung. ${ }^{5}$ With this method, uncorrelated noise, e.g. turbulent flow noise, can be rejected from the coherent sound pressure signals by using a correlated phase reference, here the speaker input.

According to Eqs. 2-5 the measured acoustic signal is a superposition of two plane waves traveling in opposite directions. The downstream and upstream traveling waves are fitted by a model proposed by Dokumaci, ${ }^{6}$ which takes losses at the wall and in the fluid into account. These losses are included in a modified wave number.

As a result of this least-mean-square fit, the four complex sound pressure amplitudes $\hat{p}_{1}^{+}, \hat{p}_{1}^{-}, \hat{p}_{2}^{+}$and $\hat{p}_{2}^{-}$ are identified at position $x=0$ for both measurements. These sound pressure amplitudes are related to each other via the reflection and transmission coefficients of the test object. This is illustrated in Figure 3 for the two different measurements $A$ and $B$. In order to calculate the reflection and transmission coefficients $r^{+}$, $r^{-}, t^{+}$, and $t^{-}$from the sound pressure amplitudes the following four relations can be derived:

$$
\begin{gathered}
\hat{p}_{1 a}^{-}=r^{+} \hat{p}_{1 a}^{+}+t^{-} \hat{p}_{2 a}^{-} \\
\hat{p}_{1 b}^{-}=r^{+} \hat{p}_{1 b}^{+}+t^{-} \hat{p}_{2 b}^{-} \\
\hat{p}_{2 a}^{+}=r^{-} \hat{p}_{2 a}^{-}+t^{+} \hat{p}_{1 a}^{+} \\
\hat{p}_{2 b}^{+}=r^{-} \hat{p}_{2 b}^{-}+t^{+} \hat{p}_{1 b}^{+}
\end{gathered}
$$

The equations from both measurements are combined and solved for the reflection

$$
r^{+}=\frac{\hat{p}_{1 a}^{-} \hat{p}_{2 b}^{-}-\hat{p}_{1 b}^{-} \hat{p}_{2 a}^{-}}{\hat{p}_{1 a}^{+} \hat{p}_{2 b}^{-}-\hat{p}_{1 b}^{+} \hat{p}_{2 a}^{-}} \quad r^{-}=\frac{\hat{p}_{2 b}^{+} \hat{p}_{1 a}^{+}-\hat{p}_{2 a}^{+} \hat{p}_{1 b}^{+}}{\hat{p}_{1 a}^{+} \hat{p}_{2 b}^{-}-\hat{p}_{1 b}^{+} \hat{p}_{2 a}^{-}}
$$

and transmission coefficients

$$
t^{+}=\frac{\hat{p}_{2 a}^{+} \hat{p}_{2 b}^{-}-\hat{p}_{2 b}^{+} \hat{p}_{2 a}^{-}}{\hat{p}_{1 a}^{+} \hat{p}_{2 b}^{-}-\hat{p}_{1 b}^{+} \hat{p}_{2 a}^{-}} \quad t^{-}=\frac{\hat{p}_{1 a}^{+} \hat{p}_{1 b}^{-}-\hat{p}_{1 b}^{+} \hat{p}_{1 a}^{-}}{\hat{p}_{1 a}^{+} \hat{p}_{2 b}^{-}-\hat{p}_{1 b}^{+} \hat{p}_{2 a}^{-}}
$$


in downstream and upstream direction, respectively. The advantage of combining the two measurements is that the resulting coefficients are independent from the reflection of sound at the duct terminations. These end-reflections are contained in the sound pressure amplitudes, but do not need to be calculated explicitly.

In order to estimate acoustic energy of the waves, we use the acoustic energy flux $I$ in a moving medium as given by Blokhintsev ${ }^{7}$ (see as well in ${ }^{8}$ ):

$$
I=\frac{1}{\rho c}(1+M)^{2}\left\langle p^{2}\right\rangle
$$

where $\langle p\rangle$ is the time-averaged acoustic pressure. Integrating over the duct cross-section area $A$ and using the pressure amplitude yields a relation between the acoustic pressure $p$ and acoustic power $P$ :

$$
P^{ \pm}=\frac{A}{2 \rho c}(1 \pm M)^{2}\left|\hat{p}^{ \pm}\right|^{2}
$$

Thereby, the energy coefficients for reflection and transmission can be given relative to the pressure coefficients.

The dissipation of acoustic energy is expressed by the dissipation coefficient. The dissipation coefficient can be calculated directly from the reflection and transmission coefficients via an energy balance:

$$
R^{ \pm}+T^{ \pm}+\Delta^{ \pm}=1
$$

The energy of the incident wave is partly reflected, partly transmitted, and partly dissipated inside the damping module. $R$ and $T$ are the power quantities of the reflection and transmission coefficients, while $r$ and $t$ have been the pressure quantities.

Assuming the same flow conditions and cross sectional area in section 1 and 2, finally, the dissipation coefficients of the test object for the downstream $\left(\Delta^{+}\right)$and upstream direction $\left(\Delta^{-}\right)$can be obtained:

$$
\Delta^{ \pm}=1-\left(\frac{(1 \mp M)^{2}}{(1 \pm M)^{2}} \cdot\left|r^{ \pm}\right|^{2}+\left|t^{ \pm}\right|^{2}\right)
$$

This is an integral value of the acoustic energy that is absorbed while a sound wave is passing the damping module. The dissipation coefficient is used to evaluate the damping performance of the test object. For most comparisons, the averaged value $\Delta_{a v g}=\Delta^{+} / 2+\Delta^{-} / 2$ is used.

\section{Test Conditions and Configurations}

The liner design was already briefly described above. In total, four FlexiS liner have been tested together with a reference liner with fully rigid cell walls. In order to enhance the inter-cell wall deflection for the FlexiS liner, certain cells have been covered with aluminium tape for the investigations presented here. Therewith, the tape covered cells behave - nearly - acoustically inactive, meaning, they are coupling only weakly with the grazing sound field of the duct. A fluctuating pressure difference on one side of the flexible cell wall is applied through the impinging sound field in the uncovered cell - forcing the wall to an increased movement. In the chosen setup every second span-wise cell row was taped (see photo in Figure 4) and ventilated by a pinhole only in order to compensate static pressure differences in the system during operation of the flow duct.

For each liner (FlexiS and reference), measurements were made for $120 \mathrm{~dB}$ and $130 \mathrm{~dB}$ overall SPL. The Mach number was varied between $M=0$ (no flow case), $M=0.1$, and $M=0.2$. These values always mark the center-line Mach number. In this paper, only data for the $130 \mathrm{~dB}$ excitation is presented.

\section{Results}

\section{A. Dissipation of Acoustic Energy}

Figure 5 shows for the taped configurations (see Figure 4) the comparison of the dissipation coefficients in the no flow case. Here, also the reference Helmholtz-Resonator liner with rigid walls (REFERENCE) was taped the same way in order to compare the same active area for all liner samples. Most of the applied flexible materials demonstrate a significant increase in the dissipation in the low frequency range from 400 to $800 \mathrm{~Hz}$. While the EP-0.5 material generates a broadening of the dissipation peak, the material 1170-0.3 


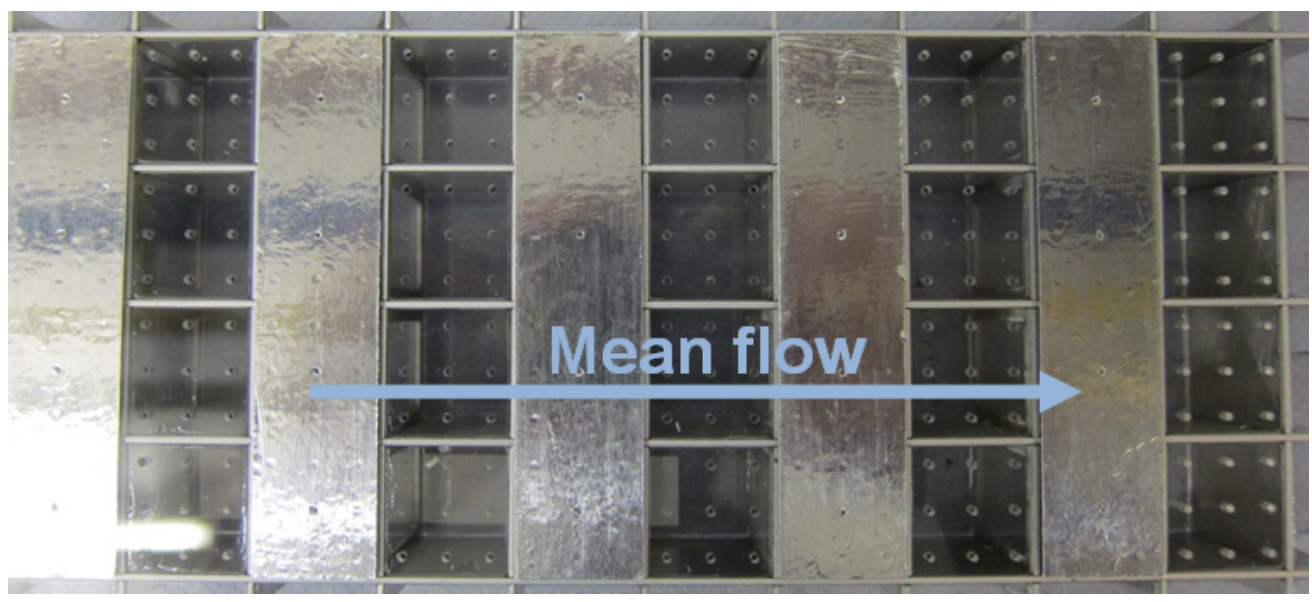

Figure 4. Photo of upper surface of FlexiS liner partly covered with tape.

shows even three dissipation maxima at 600, 1000, and $1350 \mathrm{~Hz}$. For the sample with the material 1195-0.1 the dissipation maximum is shifted from $940 \mathrm{~Hz}$ (REFERENCE) to around $750 \mathrm{~Hz}$. All FlexiS liner samples show a reduction of the maximum dissipation value. However, the integrated overall dissipation over the entire frequency range is increased. This verifies the additional damping mechanism by the deflection of the flexible walls with the intrinsic material damping characteristics.

In the case with grazing flow, the observed behavior for the averaged dissipation is comparable to the no flow case as shown in the lower two graphs of Figure 5 for a grazing flow Mach number of 0.1 and 0.2 , respectively. Here, the arithmetic average of dissipation coefficients for up- and downstream direction is displayed. In general, the grazing flow reduces the total dissipation of the liner samples. But since this affects all liner samples including the reference sample with rigid walls the relative changes in dissipation behavior are similar to the no flow case.

In order to validate the additional damping mechanism and to prove the geometric conformity of all FlexiS liners with the reference Helmholtz-Resonator liner, the samples have been tested in the non-taped configuration in another series of measurements. Hereby, as shown in Figure 6 for the no flow case, the dissipation behavior of the FlexiS liner samples (EP-0.5, 1170-0.3, 1195-0.1, 1195-0.5) with flexible walls differs only slightly from the reference Helmholtz-Resonator sample with rigid walls (REFERENCE). All liner samples indicate the same maximum dissipation for frequencies between 800 and $1000 \mathrm{~Hz}$. Only one flexible wall material (1195-0.1) with a thickness of $0.1 \mathrm{~mm}$ (green line) exhibits some dissipation increase compared to the reference sample in a frequency range between 400 and $700 \mathrm{~Hz}$.

This agreement of dissipation characteristics, which also applies for the grazing flow cases (not shown here), confirms that the phase difference between two - in propagation direction - subsequent cells is too small for an sufficient oscillation excitation of the flexible walls in between. For this reason no additional acoustic damping is achieved and the FlexiS liner samples behave like a conventional Helmholtz-Resonator liner. Only for the liner with the material 1195-0.1, a slight shift of the maximum of acoustic dissipation towards lower frequencies is observed.

\section{B. Influence of the Pinholes on the Dissipation}

The ventilation pinholes are fairly small $(0.5-1 \mathrm{~mm}$ in diameter). It is assumed, that they do not contribute significantly to the liner acoustic dissipation for most of the frequency range of interest in this study. An estimate of the resonance frequency of a covered and pinholed cell (assuming $1 \mathrm{~mm}$ pinhole) yields a resonance frequency of $280 \mathrm{~Hz}$. A validation measurement was made using pinholed cells in every second row (as in the "TAPE" measurements presented above), but with all remaining cells covered with aluminium tape too - without any pinholes for these cells. The remaining dissipation should be a good estimate for the damping effect of the pinholes and the tape covered holes itself. The results are not shown here, but an average dissipation of coefficient of 0.1 over the investigated frequency range is observed. A slight increase to maximum value of $\Delta=0.15$ at $300 \mathrm{~Hz}$ is observed for the no flow case. When a grazing flow is present, 

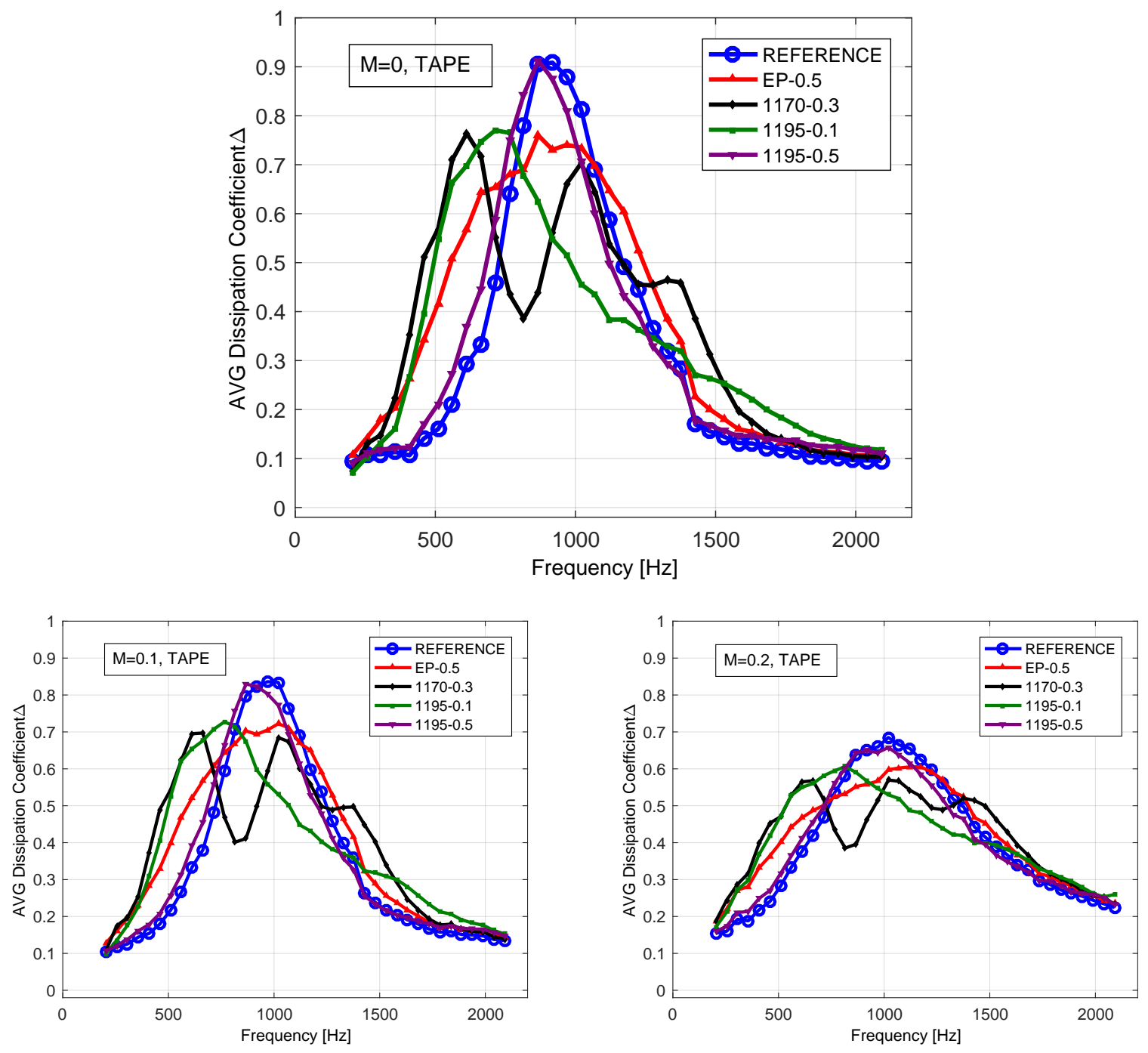

Figure 5. Averaged dissipation of acoustic energy for partly covered FlexiS liner (TAPE). Upper plot: no grazing flow $(M=0)$, lower plots: with grazing flow $M=0.1$ and $M=0.2$.

this value is decreased to $\Delta=0.1$ again.

This measurement confirms, that the observed effects in the broadening of the acoustic dissipation peaks (Figure 5) is not directly owed to the presence of an additional resonator with low frequency resonance.

\section{In-cell Measurements}

Further tests were conducted in order to better capture the behaviour of the individual cells. Three microphones were flush mounted in the back plate of three neighboring liner cells - aligned along the mean flow axis. Thereby, the pressure and phase inside the cells could be determined for the FlexiS liner and the reference liner in taped and un-taped conditions.

Figure 7 shows the sound pressure level and the phase (with respect to the microphone signal in the middle cell) of all three microphones for the standard surface ("Full" = no cells covered by tape). Generally, there are only marginal differences between all liners. The SPL in the three cells (upstream-US, mid, and downstream-DS) is almost identical. The phase shift between signals increases almost linearly with frequency which is caused by the physical distance of the cells and the decreasing wave length with increasing frequency. Near the resonance peak (around $940 \mathrm{~Hz}$ ), a common drop in phase occurs (theory suggest a nearly $180^{\circ}$ phase shift across the resonance of the cell, but this occurs for all cells). Beyond $1000 \mathrm{~Hz}$, for all liners 


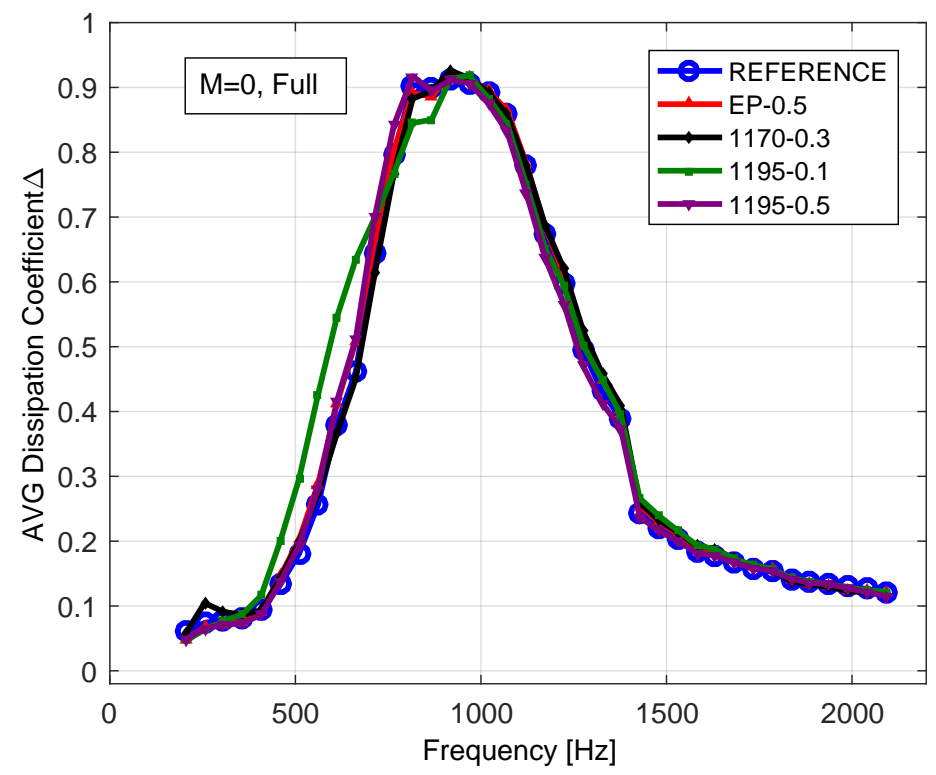

Figure 6. Averaged dissipation of acoustic energy for uncovered FlexiS liner (FULL). No grazing flow (M=0).

an almost linear increase can be observed again. Only for the material 1195-01, where also an increase in dissipation was observed for the range from 400-700 Hz (compare Figure 6), the intermediate drop in phase shift occurs for a lower frequency (around $700 \mathrm{~Hz}$ ). This suggests, that the resonance of the cells is shifted towards lower frequencies by the $0.1 \mathrm{~mm}$ thin TPU sheet cell wall.

In Figure 8, the sound pressure level and the phase for the taped configurations are shown. Here, the upstream and downstream cells were covered by tape with only the small pinhole for ventilation of mean pressure in the surface. The middle cell was not covered and fully exposed to the grazing sound field.

For the reference liner with rigid walls, the sound pressure in the mid cell is much higher than for the other two cells over the entire frequency range. The small differences below $800 \mathrm{~Hz}$ might be owed to nonsymmetrical pinholes in the upstream (US) and downstream (DS) cells. The phase difference for US and DS cells coincide for a frequency of $204 \mathrm{~Hz}$. Using a simple 1-D acoustic network model and assuming a pinhole of $0.5 \mathrm{~mm}$ diameter (tape thickness also $0.5 \mathrm{~mm}$ ), and taking the further geometry of face sheet and cell into account, a phase difference for an acoustic wave entering the covered cell compared to the open middle cell of around $50^{\circ}$ is obtained. However, this value strongly depends on the geometry of the pinhole, as it is coupled to the low frequency resonance of the covered cell (compare sec. B). Above $1000 \mathrm{~Hz}$, where the phase shift for the middle cell occurs, the canonical behavior of "early arrival" of the signal at the upstream cell and "late arrival" at the downstream cell can be observed, meaning that the higher frequencies can enter the cell via the pinhole, although, as visible from the SPL plot, strongly damped. For the reference liner, there is no interaction between neighboring cells across the cell walls.

For the FlexiS liner, the behavior is different. Looking at the SPL curves, only for the EP-0.5 material some larger difference between the covered and uncovered cells are visible below $1200 \mathrm{~Hz}$. The thicker material $(0.5 \mathrm{~mm})$ and higher intrinsic damping allows only little communication between neighboring cells and yields a behaviour nearly like a rigid wall (offset of phase for low frequency, strong difference in SPL over nearly the entire frequency range of investigation).

For the other two materials (1170-0.3 and 1195-0.1), there are deviations in the SPL only for higher frequencies. That means, that via a deflection of the flexible walls a high SPL is generated also in the covered cells with only frequencies above $1200 \mathrm{~Hz}$ not being able to produce significant deflections. Considering the small axial distance between neighboring cells $(20 \mathrm{~mm}$ from one to the next), a covered cell will be subject to nearly simultaneous pressure increase from both neigbouring open cells, and subsequently simultaneous decrease again in the considered frequency range. Thus, the SPL in the covered cells are mainly determined by the uncovered cells. 

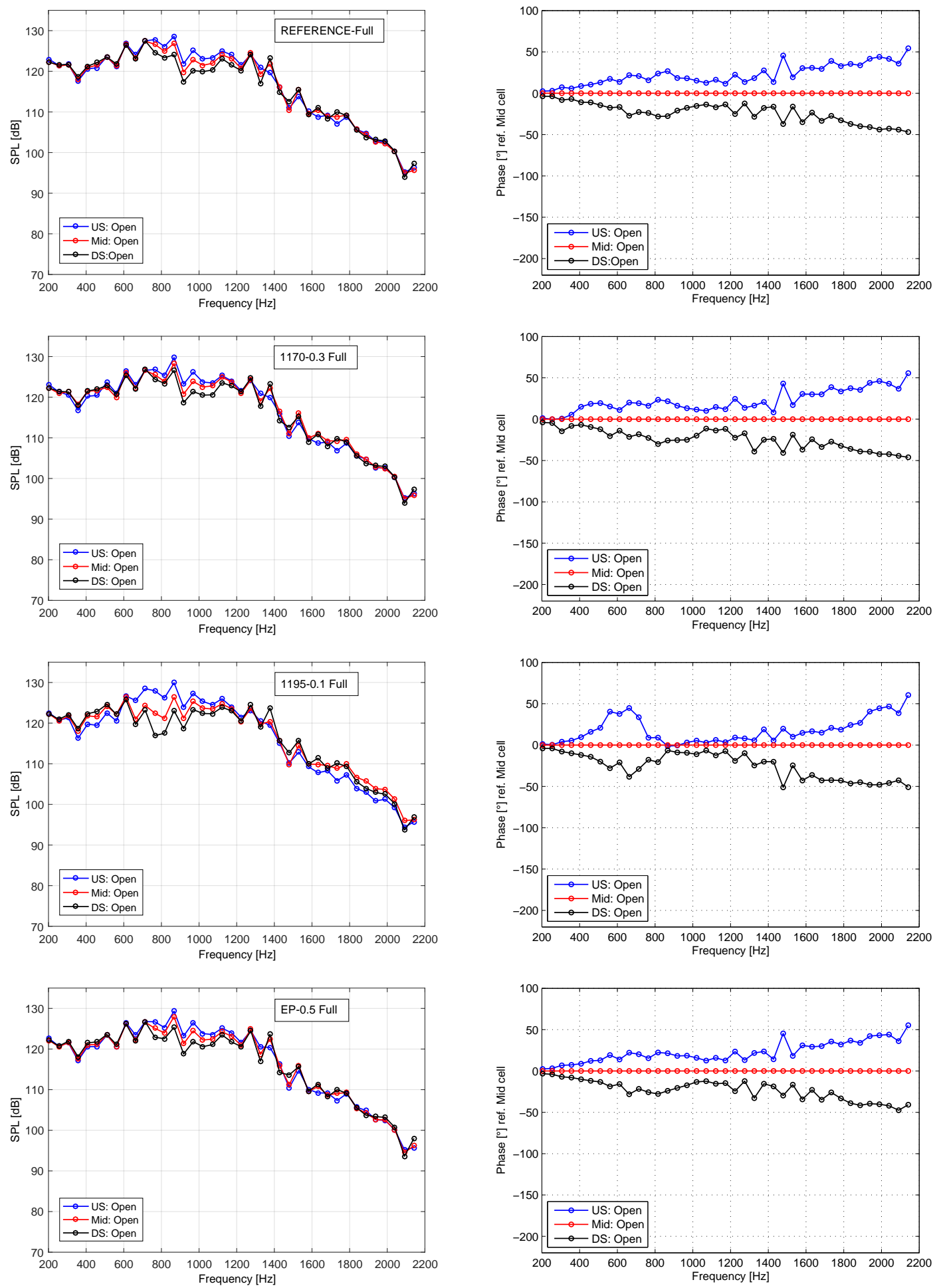

Figure 7. Sound pressure level inside the cells and phase angle with respect to microphone in mid cell: comparison of reference liner to FlexiS liner, full liner surface active. No grazing flow $(\mathrm{M}=0)$. 

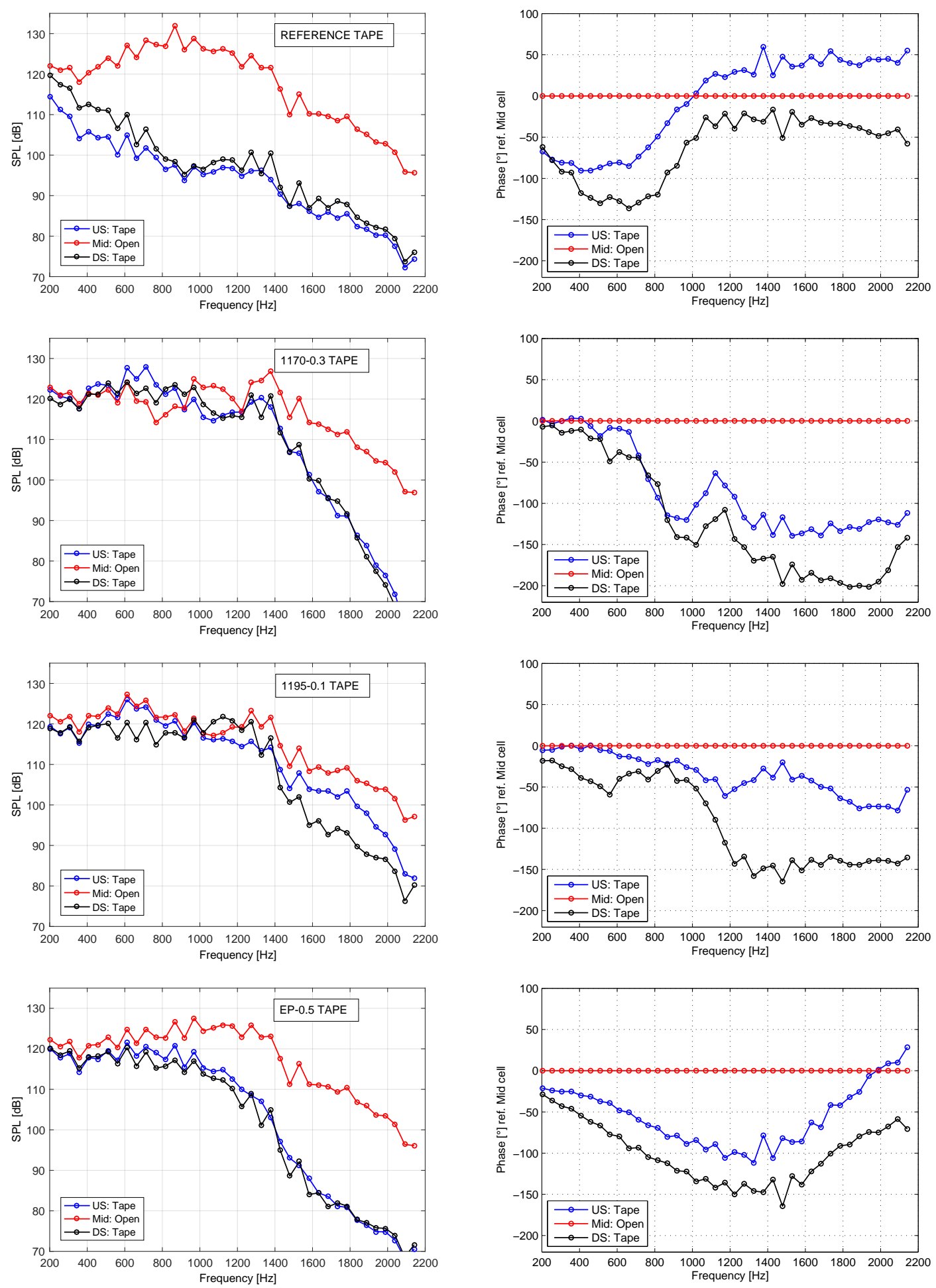

Figure 8. Sound pressure level inside the cells and phase angle with respect to microphone in mid cell: comparison of reference liner to FlexiS liner, every second row of liner surface covered (TAPE). No grazing flow $(M=0)$. 
It is rather difficult to interpret the phase information for these two configurations in detail. For low frequencies, the direct coupling of open and covered cells via the flexible cell walls is directly visible through the zero/low phase difference between neighboring cells. The expected divergence for upstream and downstream cell for higher frequencies can be well observed. Changes in the slope of the phase curve may relate to resonances in the cavity (and/or the flexible wall) which manifests in (local) dissipation maxima (Figure 5).

The presented results reveal the enhanced damping potential of the FlexiS liner concept with flexible wall structures and additional material damping. This concept allows to broaden the effectively damped frequency range and to shift the damping characteristics to lower frequencies without increasing the liner size and without the demand of additional energy. Compared to a "normal" double-degree-of-freedom (DDOF) liner, the effect of the intrinsic damping of cell walls is added. In addition, future investigations and applications may contain the coupling of the in-active parts (the covered cells in the current investigation) to multiple active cells.

\section{Conclusions and Outlook}

In this experimental investigation, a novel liner concept (FlexiS) has been introduced and tested. An increased overall damping performance and an increased frequency range of significant damping mainly at lower frequencies could be obtained by replacing some of the rigid walls of an SDOF liner by flexible materials with inherent damping. The concepts proved its benefits if some non-active parts (i.e. no direct connection to the grazing sound field) were placed between the active cells. Measurements of the dissipated acoustic energy of the whole liner and of the acoustic pressure inside neighboring cells reveal the importance of the flexible walls. In the current investigation, the arrangement of active (open) and non-active (taped) cells allowed for a great flexibility in liner setup without comprehensive changes or re-manufacturing of the liner. However, in terms of an efficient damping of acoustic energy, this setup represents a loss in active damping surface. Therefore, future works needs to address the influence and requirements for the non-active parts (required size, coupling between neighboring non-active cavities, etc.) in order to find an efficient arrangement and investigate the actual deflection of the flexible walls with the final aim of modeling liners with flexible structures accurately.

\section{Acknowledgments}

The financial support of the work in the framework of the LuFo V-2 project LaKS ("Laermabsorbierende Kunststoff-Strukturen/Noise Absorbing Composite Structures") by the Federal Ministry for Economic Affairs and Energy (contract number 20E1502A) based on a decision of the German Bundestag is gratefully acknowledged. The authors would like to thank all partners of the project (Fraunhofer IAP/PYCO, TU Dresden, BTU Cottbus-Senftenberg, and TU Berlin) for the various contributions which finally enabled the acoustic investigations presented here.

\section{References}

\footnotetext{
${ }^{1}$ von Helmholtz, H., Die Lehre von den Tonempfindungen als physiologische Grundlage für die Theorie der Musik (On the sensations of tone as a physiological basis of the theory of music), Vieweg, Braunschweig, 1863.

${ }^{2}$ BASF, "Thermoplastic Polyurethane Elastomeres," Elastollan ${ }^{\circledR}$ - Material Properties, issued September 2017, accessed online at http://www.polyurethanes.basf.de/pu/Elastollan/Elastollan_Materialeigenschaften on 07 November 2017.

${ }^{3}$ Busse-Gerstengarbe, S., Bake, F., Enghardt, L., and Jones, M. G., "Comparative Study of Impedance Eduction Methods, Part 1: DLR Tests and Methodology," 19th AIAA/CEAS Aeroacoustics Conference, No. 2013-2124, Berlin, Germany, May 2013.

${ }^{4}$ Lahiri, C., Sadig, S., Gerendas, M., Enghardt, L., and Bake, F., "Establishment Of A High Quality Database For The Modelling Of Perforated Liners," Journal of Engineering for Gas Turbines and Power, Vol. 133, No. 9, September 2011, pp. 091503-1 - 091503-9.

${ }^{5}$ Chung, J. Y., "Rejection of flow noise using coherence function method," Journal of the Acoustical Society of America, Vol. 62, 1977, pp. 388-395.

${ }^{6}$ Dokumaci, E., "A note on transmission of sound in a wide pipe with mean flow and viscothermal attenuation," Journal of Sound and Vibration, Vol. 208, No. 4, 1997, pp. 653-655.

${ }^{7}$ Blokhintsev, D. I., Acoustics of a nonhomogeneous moving medium, NACA, Technical Memorandum 1399, 1946.

${ }^{8}$ Morfey, C. L., "Acoustic energy in non-uniform flows," Journal of Sound and Vibration, Vol. 14, No. 2, 1971, pp. 159-170.
} 